\title{
Indústria transformadora portuguesa: fatores determinantes da entrada de novas empresas
}

\author{
Portuguese manufacturing industry: determinants of new firm entry
}

\author{
Georgette Andraz \\ Universidade do Algarve, Escola Superior de Gestão, Hotelaria e Turismo, Campus da Penha, 8005-140 Faro, Portugal, \\ gandraz@ualg.pt
}

\section{J. Sant'ana Fernandes}

Universidade do Algarve, Escola Superior de Gestão, Hotelaria e Turismo, 8005-140 Faro, Portugal, jsfer@ualg.pt

\section{Cristina Gonçalves}

Universidade do Algarve, Escola Superior de Gestão, Hotelaria e Turismo, 8005-140 Faro, Portugal, cjesus@ualg.pt

\section{J. Rodrigo Guerreiro}

Universidade do Algarve, Escola Superior de Gestão, Hotelaria e Turismo, 8005-140 Faro Portugal, jrodrigo@ualg.pt

\section{Resumo}

0 presente estudo investiga os fatores que influenciam a entrada de novas empresas na indústria transformadora portuguesa no período de 1996-2007. Confirma-se de uma forma global as conclusões de estudos anteriores, designadamente Fotopoulos e Spence (1998), Nystrom (2007), entre ouros. A dimensão do mercado e as condições macroeconómicas do período anterior são fatores importantes na decisão de entrada. 0 estudo confirma ainda uma forte relação positiva entre entradas e saídas de empresa do período anterior. Foram encontradas evidências de que as barreiras à entrada são pouco significativas. Este facto associado à elevada rotação empresarial sugere uma fraca capacidade de adaptação das empresas ao mercado, designadamente pela sua incapacidade de realizar os investimentos necessários para a sobrevivência e crescimento. Os resultados sugerem ainda uma relação não linear, na forma de "U" invertido, entre lucros da indústria e novas entradas, facto que introduz uma nova linha de investigação sobre o tema.

Palavras-chave. Dinâmica empresarial, entrada, custos irreversíveis, indústria transformadora, barreiras à entrada e saída.

\begin{abstract}
The main purpose of this study is to investigate the determinants of new firm entry in the Portuguese manufacturing industries over the period 1996-2007. Estimation results generally confirm previous studies findings (see, e.g. Fotopoulos \& Spence, 1998; Nystrom, 2007). Our findings indicate that market size and past macroeconomic conditions are important determinants of entry rates. Results also indicate a significant positive effect of past exit on actual entry. It is also found evidence of low barriers to new entry which associated with high firm turnover suggests companies' inability to compete in the market, mainly due to difficulties in raising capital for required investments to operate efficiently. Results also reveal evidence of an inversed " $U$ " shaped relationship between industry profit and entry. This result brings new line of investigation in the empirical literature on industry dynamics.
\end{abstract}

Keywords: firm dynamics, entry, exit, manufacturing industry, barriers to entry and exit.

\section{Introdução}

0 processo de entrada de novas empresas tem sido objeto de interesse teórico e empírico na literatura económica, ao longo das últimas décadas. Fundamenta-se o interesse por este fenómeno com o pressuposto de que a entrada, assim como a saída de empresas estabelecidas, é fator importante da competitividade e promoção da eficiência dos mercados. Segundo a abordagem de Schumpeter (1935) a pressão competitiva das novas empresas mais eficientes elimina do mercado as menos eficientes. Este processo melhora a eficiência dos mercados, acelera o processo de criaçãodestruição, frequentemente entendido como um prérequisito inevitável da seleção e evolução dos mercados.

As primeiras investigações sobre a dinâmica empresarial, assentes na abordagem da organização industrial versaram sobre a criação de empresas e seus fatores determinantes. Segundo esta abordagem, a entrada de novas empresas está positivamente relacionada com as expectativas de benefícios após a entrada. Sustenta também que os benefícios a longo prazo de uma indústria dependem, em grande medida, das barreiras que limitam a intensidade da competição e criam condições para as empresa estabelecidas aumentarem os preços e obter lucros anormais. Na sequência das teses propostas por Bain (1956) a existência de barreiras à entrada depende de duas condições: (1) a presença de condições estruturais, tais como: economias de escala, a diferenciação do produto e as vantagens absolutas de custos das empresas estabelecidas e (2) o comportamento das empresas estabelecidas em explorar estas condições na presença de entradas potenciais e atuais.

Nesta perspetiva, a entrada de novas empresas está positivamente relacionada com as expectativas de lucros de mercado e inversamente relacionada com as barreiras à entrada.

O modelo tradicional da organização industrial sobre as barreiras que limitam o processo de entrada de empresas 
está teoricamente associado a Bain (1956) e empiricamente a Geroski (1991; 1991a) e Orr (1974).

Todavia, estudos empíricos têm mostrado que podem ocorrer entradas mesmo quando a rendibilidade da indústria é nula (Baldwin, 1995). Por outro lado, de acordo com diversos autores, designadamente Segarra (2002), apesar da existência de barreiras à entrada que limitam o fluxo de entradas na indústria, em geral, as taxas de entrada sectoriais são elevadas.

Segundo abordagens mais recentes, nomeadamente, Geroski (1991) a entrada de novas empresas depende não só das expectativas dos lucros após entrada mas também de outros fatores estruturais, da indústria, do ciclo de vida da indústria, do comportamento das empresas em atividade, nomeadamente quanto ao seu potencial de inovação, das condições económicas, entre outros, tendo como obstáculos as barreiras endógenas e exógenas à entrada.

Não obstante a extensa literatura empírica existente sobre o tema em apreço, a evidência empírica e as teorias da economia industrial nem sempre são consensuais (Geroski, 1995). Esta circunstância poderá indicar que é possível um conhecimento mais sistemático do processo da dinâmica empresarial e dos fatores que a determinam, particularmente a entrada de novas empresas no contexto da indústria portuguesa.

Neste contexto, este estudo tem como objetivo principal analisar os determinantes da entrada de novas empresas na indústria transformadora portuguesa. Esta análise deverá dar reposta às seguintes questões: Quais os fatores que motivam ou condicionam a decisão dos agentes económicos de entrar nos mercados em diferentes sectores da indústria transformadora? As características estruturais, estratégicas da indústria, variações macroeconómicas e a evolução cíclica da indústria são importantes para explicar os padrões de entrada na indústria?

As respostas a estas questões poderão ser importantes para a definição de políticas que estimulem a sobrevivência das empresas e, deste modo, contribuam para minimizar as taxas de desemprego que se tem vindo a registar nos últimos anos.

No contexto dos objetivos gerais do estudo formula-se as seguintes hipóteses de investigação:

Hipótese I - O comportamento de entrada no mercado está negativamente relacionado com vetor de barreiras à entrada.

Hipótese II - O comportamento de entrada no mercado está positivamente relacionado com vetor de variáveis relativas a incentivos ou oportunidades de mercado.

Este trabalho desenrola-se em quatro partes distintas. A próxima secção apresenta uma breve revisão da literatura teórica e empírica dos fatores determinantes de entrada, a secção três expõe a metodologia utlizada no estudo. A quarta secção apresenta os resultados da estimação e a última apresenta as principais conclusões e considerações sobre políticas públicas.

\section{Breve revisão da literatura}

Esta secção apresenta uma breve revisão sobre os fatores que podem influenciar a entrada de novas empresas. Apresenta-se também resultados de estudos empíricos anteriores. 0 modelo teórico subjacente aos fatores que influenciam a decisão de entrada é apresentado posteriormente.

A literatura teórica e empírica sobre a dinâmica empresarial distingue os determinantes do comportamento de entrada, também designado na terminologia de Bain (1956) como determinantes "da condição de entrada", em duas categorias: primeiro, os incentivos, designadamente a dimensão do mercado $\mathrm{e}$ as expectativas de lucros e segundo, o convencionalmente considerado barreiras à entrada.

A maioria da literatura que analisa as barreiras, no sentido em que dificultam a entrada de novas empresas, tem por base os trabalhos pioneiros de Orr (1974) e Geroski (1991).

\section{Incentivos}

A literatura empírica sobre a dinâmica empresarial utiliza três grupos de incentivos à entrada: lucros ou rendibilidade histórica, dimensão da indústria e abandono ou saída de empresas estabelecidas.

De uma forma geral, os resultados de estudos empíricos sobre determinantes de novas entradas não são inequívocos. Por exemplo, evidencia-se que a maioria dos resultados relativos a mais de 70 estudos empíricos de 11 países, resumido por Siegfried e Evans (1994), encontra uma forte correlação positiva entre a rendibilidade histórica da indústria, dimensão do mercado e novas entradas.

Todavia, outros estudos, designadamente de Mata (1993; 1996), Nystrom (2007) e Andraz (2013) não confirmam a relação positiva e significativa entre aquelas variáveis e novas entradas.

\section{Barreiras à entrada}

Na sequência das teses propostas por Bain (1956), a existência de barreiras à entrada depende de duas condições: (1) barreiras estruturais tais como economias de escala, a diferenciação do produto e as vantagens absolutas de custos das empresas estabelecidas e (2) barreiras estratégicas.

As maiores contribuições nesta área de investigação estão associadas a Caves e Porter (1976) e Yip (1982).

Esta secção faz uma breve revisão dos fatores que constituem barreiras estruturais, na perspetiva de Bain (1956), bem como outras fontes de barreiras incluídas noutros estudos empíricos, nomeadamente Barbosa (2007), sobre o tema e utilizados neste trabalho.

\section{Barreiras estruturais}

A presença de economias de escala pode constituir uma barreira à entrada quando a dimensão mínima de eficiência do mercado é relativamente elevada, forçando entradas com dimensão suficiente que permita obter as vantagens das empresas já estabelecidas. Esta condição de entrada pode desincentivar novas entradas. De uma forma geral, os 
resultados empíricos sobre diversos países resumidos por Siegfried e Evans (1994) não são conclusivos.

Modelos da organização industrial desenvolvidos por diversos autores, designadamente Lambson (1991), têm subjacente o princípio de que os custos irreversíveis aumentam os custos de entrada (e simetricamente de saída) e criam uma zona de inação onde a probabilidade de entrada e saída de empresas estabelecidas é reduzida.

O estudo empírico aplicado à indústria transformadora portuguesa de Mata (1991) sugere que os custos irreversíveis constituem barreiras importantes apenas para as grandes empresas. No estudo empírico aplicado à indústria transformadora dos EUA (Kessides, 1991) concluiu-se que a influência dos custos irreversíveis sobre as entradas de novas empresas é significativa e negativa. Resultados do estudo de Mata (1991), seccionado de acordo com a dimensão de entrada, da indústria transformadora portuguesa no período de 1979-1982, sugerem que os custos irreversíveis constituem barreiras importantes apenas para as grandes empresas, enquanto Andraz (2013) não encontrou evidências de uma relação entre custos irreversíveis e novas entradas.

A concentração da indústria é uma das variáveis estruturais mais utlizadas na literatura empírica. Associada ao poder de monopólio das empresas instaladas representa, por isso, uma ameaça competitiva (significativa) para potenciais novas entradas, reduzindo as suas possibilidades de sobrevivência (Nystrom, 2007). Resultados empíricos sobre o impacto da concentração também não apresentam um padrão de resultados consistente (Geroski, 1995).

Relativamente aos requisitos/necessidade de capital a hipótese evidenciada na literatura é que as empresas estabelecidas têm maior capacidade de obtenção de recursos junto do mercado de capitais. Utilizando como medida a intensidade de capital físico, alguns estudos designadamente Khemani e Shapiro (1987), Mata (1993) e Fotopoulos e Spence (1998) sugerem que elevadas necessidades de capital proporcionam fortes barreiras à entrada. Por outro lado, a evidência empírica de Austin e Rosenbaum (1990) e Siegfried e Evans (1994), entre outros, sugere que a entrada de novas empresas no mercado não é substancialmente dificultada em indústrias de capital intensivo.

Produtividade do trabalho é entendida como forte barreira a novas entradas na medida em que níveis relativamente elevados de produtividade do trabalho estão frequentemente associados a elevados investimentos em capital tangível e intangível e elevado desempenho competitivo das empresas estabelecidas. Estes investimentos representam fontes potenciais de barreiras e, nesta medida, desencorajam novas entradas (Taymaz, 1997).

\section{Barreiras estratégicas}

A literatura teórica e empírica apresenta duas abordagens sobre a relação entre a dinâmica industrial e intensidade tecnológica da indústria. A primeira, sugerida por contributos teóricos de Bain (1956) e Yip (1982) e por estudos empíricos, nomeadamente de Orr (1974), evidencia a inovação como barreira à entrada. A segunda abordagem, desenvolvida a partir de evidência empírica e modelos teóricos alternativos, é sugerida por alguns autores, nomeadamente Acs e Audretsch (1989), argumenta que o ambiente tecnológico proporciona um veículo adequado para novas entradas.

As duas explicações teóricas sobre a relação entre entradas e intensidade de despesas em investigação e desenvolvimento (I\&D) proporcionam uma visão confusa que corresponde de algum modo às conclusões, por vezes contraditórias, da evidência empírica disponível até à data.

O capital humano qualificado e a existência de empresas multi estabelecimentos são fatores normalmente considerados como barreiras estratégicas na literatura empírica. Diversos estudos empíricos, designadamente Geroski, Mata e Portugal (2007), Barney (1991) e Khemani e Shapiro (1987), mostram que estes fatores permitem às empresas desenvolver capacidades específicas e consequente contribuição para a obtenção de vantagens no mercado, representando, deste modo, forte barreira a novas entradas.

\section{Variáveis de conjuntura}

Teoria e estudos empíricos, na perspetiva da organização industrial (Klepper,1996) permitiram definir um conjunto de regularidades sobre o ciclo de vida das indústrias, sugerindo que novas entradas e inovação dependem do grau de maturidade da indústria. Constatam-se elevados níveis de entrada nas fases iniciais e redução de entradas e alteração da natureza de inovação à medida que se atinge a fase de maturidade. Nesta fase, a entrada de novas empresas diminui significativamente, refletindo um período de reestruturação do sector (com taxas líquidas de entradas negativas. A terceira fase do ciclo de vida é caracterizada pela estabilização da população de empresas (com entradas próximas de zero).

A literatura teórica refere duas hipóteses que sustentam a influência macroeconómica sobre o fluxo de novas entradas. A primeira argumenta que condições económicas favoráveis propiciam a criação de oportunidades de negócios e favorecem a sobrevivência de novas empresas. A entrada é nesta perspetiva, um fenómeno pró - cíclico. A hipótese alternativa assume que um crescente número de empresas podem ser criadas quando os custos de oportunidade de ser empreendedor são baixos, isto é, em períodos de elevado desemprego. Esta hipótese corresponde à visão da entrada como um fenómeno contra cíclico. Apesar da reconhecida importância das condições cíclicas dos negócios sobre a dinâmica empresarial a evidência empírica é inconclusiva.

Em Portugal, Andraz (2013) e Mata (1996) concluíram que a criação de novas empresas na indústria transformadora tem um comportamento pró-cíclico, enquanto Wagner (1994), num estudo semelhante aplicado à indústria transformadora alemã, não encontrou evidência confirmando qualquer hipótese.

\section{Metodologia}

A análise empírica deste estudo assenta fundamentalmente em três fontes estatísticas, permitindo a validação cruzada e a complementaridade de informação: os Quadros de Pessoal (QP), recolhidos pelo Gabinete de Estratégia e Planeamento do Ministério do Trabalho e da Solidariedade Social (GEP/MTSS), 
o Inquérito às Empresas Harmonizado (IEH), produzido pelo Instituto Nacional de Estatísticas (INE) e dados relativos à atividade de I\&D, produzido pelo GPEARI do Ministério da Ciência, Tecnologia e Ensino Superior (MCTES).

A classificação bem como o nível de desagregação dos sectores seguiu a adotada pelo INE - Estatísticas IEH, uma vez que era necessário uma plataforma de harmonização que permitisse agregar os dados das duas fontes utlizadas (QP e INE). Foram identificadas um conjunto de limitações no processo de compatibilização dos ficheiros de dados destas duas fontes, também identificadas por outros autores nomeadamente Carreira (2004) e Mata (1991). Por forma a minimizar a ausência de informação económica e financeira das empresas com menos de dez trabalhadores, à semelhança de outros estudos, nomeadamente Mata (1991), foram eliminados da base de dados a classe de entidades com menos de nove trabalhadores ao serviço.

As hipóteses de pesquisa foram testadas num painel de dados relativos ao período de 1996 a 2007, considerando os 262 sectores a 5 dígitos (divisão da classificação da atividade económica - CAE), posteriormente agregados a 20 setores a 2 dígitos da CAE.

De acordo com a metodologia Eurostat (2006), a contagem das empresas novas num dado sector de atividade considerou o seu número de registo. Considera-se entrada do ano t quando o número de identificação de uma entidade é superior ao último número existente no ficheiro do ano t-1.

Os encerramentos ou saídas ocorrem quando estas deixam de estar presentes nos ficheiros da base de dados, pelo menos durante dois anos consecutivos, ou quando passam a ter menos de nove trabalhadores ao serviço. As empresas estabelecidas na população ativa foram definidas como as existentes na base de dados do ano t.

Os dados recolhidos têm uma estrutura de dados em painel, equilibrado, ou seja com igual número de observações por categoria/ano.

As estimações foram desenvolvidas pelos seguintes modelos econométricos: Mínimos quadrados ordinários (modelo pooled), painel de efeitos fixos e painel de efeitos aleatórios. A realização de um conjunto de testes (teste $\mathrm{F}$, teste BreuschPagan e teste de Hausman) indicados na literatura econométrica existente sugeriu o modelo fixo como o mais indicado para a estimação da equação de entradas. Porém, o emprego deste estimador (efeitos fixos) tem como desvantagem barrar as variáveis que não variam ao longo do período do estudo (Hsiao, 2003). Na sequência desta limitação, não foram estimadas variáveis que traduzem características estruturais e estratégicas da indústria, respetivamente, ciclo de vida e intensidade tecnológica.

\section{Modelo e variáveis}

Para analisar a influência sobre a entrada das variáveis explicativas mais utlizadas na literatura utilizou-se uma especificação econométrica conceptualmente similar ao modelo estático inicialmente apresentado por Orr (1974) ao qual Geroski (1991) introduziu outras evoluções decorrentes de outras abordagens, nomeadamente a evolucionária (Nelson e Winter 1982), e a do ciclo de vida (Klepper, 1996).

A função adotada para estimar os efeitos dos fatores sobre novas entradas tem como variável dependente o logaritmo (neperiano) do número absoluto de entradas e as variáveis explicativas são os fatores determinantes de novas empresas na indústria - representada pela letra $\mathrm{i}$ - durante o período - prescrita pela t. Foram testadas equações com variável dependente medida em termos de valor absoluto de entrada, taxa de entrada e os seus logaritmos - logaritmo do número absoluto de entrada e taxa de entrada. Desde logo, os resultados da estimação dos modelos sugeriram uma maior sensibilidade utilizando a transformação logarítmica do número absoluto.

A definição das variáveis e sinais esperados segue a abordagem de Fotopoulos e Spence (1998) e Shapiro e Khemani (1987). Neste estudo, assumimos que a decisão de entrada é resultado das observações da estrutura do período atual para todas as variáveis com exceção da saída de empresas, a dimensão do mercado e a taxa de crescimento do PIB referidas ao período anterior ( $\mathrm{t}-1$ ).

De acordo com análise realizada, no presente estudo são consideradas as seguintes variáveis agrupadas em quatro vetores: (i) estruturais; (ii) estratégicas; (iii) cíclicas; (iv) interação. Na estimação foi considerada também a forma quadrática das variáveis rendibilidade (Lu2) e intensidade capitalística (ICtb2.it) para captar a possibilidade da não linearidade da relação entre estas variáveis e a entrada.

Tanto quanto tenhamos conhecimento, nenhum outro estudo sobre o tema analisou as relações interação das variáveis ou as relações não lineares aqui estudadas.

Neste sentido, a forma funcional da função apresenta-se como se segue:

$$
\begin{aligned}
& L E N T 01_{i t}=\alpha_{i}+\beta_{1} L u_{i_{t}},+\beta_{2} L u 2_{i_{t}}+\beta_{3} D m_{i t}+\beta_{4} \operatorname{LSAI}_{i_{t-1}}+\beta_{5} D I M E_{i t},+\beta_{6} I_{C t} b_{i_{t}},+\beta_{7} I C t b 2_{i_{t}},+ \\
& \beta_{8} \text { Pd }_{i_{t}},+\beta_{9} \text { LEst }_{i_{t}}, \beta_{10} \text { Itec }_{i},+\beta_{11} \text { Habi it }_{i_{t}},+\beta_{12} \text { PIB }_{t-1}+\beta_{13} \text { CicloC }_{i},+\beta_{14} \text { CicloI }_{i}+\beta_{15} \text { Litec }_{i_{t}},+ \\
& \beta_{16} \mathrm{ITPd}_{i_{t}}+\beta_{17} I C C i_{i_{t}},+\beta_{18} L h h i_{i_{t}}+\mu_{i_{t}}
\end{aligned}
$$

Onde LENT01i é a variável dependente e representa a entrada de novas empresas na indústria.

As tabelas que seguem mostram a definição das variáveis utilizadas na análise empírica, suas medidas, sinal esperado, de acordo com a revisão da literatura apresentada anteriormente, e respetivas fontes estatísticas.

Na tabela 1 estão apresentadas as proxies de incentivos das quais se espera, em geral, uma relação positiva com as entradas de novas empresas. Na variável lucro assume-se a possibilidade de uma relação não linear, motivada por resultados contraditórios observados em estudos anteriores. Não foi encontrada na literatura, até à data, outro estudo que tenha analisado a hipótese da não linearidade desta relação, pelo que não se avança um sinal esperado à priori, pelo que assinalamos na tabela com os sinais (+-). 
Tabela 1 - Incentivos

\begin{tabular}{|l|c|c|l|c|c|}
\multicolumn{1}{|c|}{$\begin{array}{c}\text { Estruturais }- \\
\text { Incentivos }\end{array}$} & Nome & Sinal & \multicolumn{1}{c|}{ Definição } & Fonte \\
\hline Lucros & $\mathrm{Lu}$ & + & $\begin{array}{l}\text { Resultado liquido mais impostos/ Numero de empresas estabelecidas no } \\
\text { período t }\end{array}$ & INE \\
\hline Lucros2 & $\mathrm{Lu} 2$ & $+/-$ & Quadrado do rácio: (Resultado líquido mais impostos/N_empresas) & INE \\
\hline Dimensão de mercado & $\mathrm{Dm}$ & + & Quota de mercado da indústria/emprego total da economia no período t-1. & QP/INE \\
\hline Saídas b) & $L S A I_{t-1}$ & + & $\begin{array}{l}\text { Logaritmo do número absoluto de saídas do período t-1 } \\
\text { LnSai =0 se Sai=0 = Ln (Sai+0.1) se Ent } \neq 0\end{array}$ & QP \\
\hline
\end{tabular}

Fonte: GPEARI-Gabinete de Planeamento, Estratégia, Avaliação e Relações Internacionais; INE- Instituto Nacional de Estatística; QP- Quadros de Pessoal do MTSS; MTSS- Ministério do Trabalho e Solidariedade Social.

Foram testadas duas medidas proxies de rendibilidade/lucro da indústria: a rendibilidade das vendas (valor acrescentado médio anual da indústria menos custos com pessoal a dividir pelas vendas) e rendibilidade medida em termos de resultados líquidos. Esta última pareceu refletir melhor o atrativo do mercado para novos capitais. Todas as variáveis são referidas ao período t, exceto a dimensão do mercado e saídas, ambas referidas ao período anterior.

Tabela 2 - Barreiras estruturais

\begin{tabular}{|c|c|c|c|c|}
\hline Barreiras & Nome & Sinal & Definição & Fonte \\
\hline \multicolumn{5}{|l|}{ Estruturais } \\
\hline Economias de Escala & DIME & $-/+$ & $\begin{array}{l}\text { Emprego médio das empresas com dimensão mínima eficiente } \\
\text { (DME) } \\
\text { (Rácio entre emprego das empresas com DME (t) e o emprego } \\
\text { da indústria do período t-1) }\end{array}$ & QP \\
\hline $\begin{array}{l}\text { Produtividade do } \\
\text { trabalho }\end{array}$ & $\mathrm{Pd}$ & $+/-$ & $\begin{array}{l}\text { Logaritmo (valor acrescentado bruto a preços de mercado/ } \\
\text { trabalhadores) }\end{array}$ & INE \\
\hline Intensidade de capital & ICtb2 & $+/-$ & Quadrado do rácio: (Imobili_corpóreo /trabalhador) & INE \\
\hline $\begin{array}{l}\text { Intensidade de capital } \\
\text { (proxy de custos } \\
\text { irreversíveis) }\end{array}$ & ICtb & - & Imobili_corpóreo /trabalhador & INE \\
\hline Concentração & Lhhi & - & $\begin{array}{l}\text { Logaritmo da Concentração do sector (Índice de Hirschmann) - } \\
\text { Somatório do quadrado da participação de cada empresa em } \\
\text { relação ao total do emprego da indústria no período } t \text { (varia entre } \\
0 \text { e 1.000). }\end{array}$ & QP \\
\hline \multicolumn{5}{|l|}{ Estratégicas } \\
\hline $\begin{array}{l}\text { Multi- } \\
\text { estabelecimentos }\end{array}$ & LEst & - & $\begin{array}{l}\text { Logaritmo do número médio de estabelecimentos por empresa do } \\
\text { período anterior. }\end{array}$ & $\mathrm{QP}$ \\
\hline Intensidade tecnológica & $\begin{array}{l}\text { Intens_tecn_alta (ItecA) } \\
\text { Intens_tecn_med (ItecM) }\end{array}$ & $+/-$ & Despesas em I\&D /vendas & GPEARI \\
\hline Habilitações superiores & Habi & - & Trabalhadores com formação superior/Total trabalhadores & QP \\
\hline
\end{tabular}

Para algumas variáveis não se assume, à priori, um tipo específico de relação e assinalamos na tabela com os sinais (+-).

Na tabela 3 estão apresentadas um conjunto de variáveis de interação. A introdução de efeitos de interação destas variáveis tem natureza exploratória e pretende captar relações mútuas sobre a variável dependente, evidenciando que o efeito produzido por uma determinada variável explicativa está associado ao nível (valor) em que se
Na tabela 2 estão apresentadas as proxies de barreiras à entrada, agrupadas em estruturais e estratégicas. De uma forma geral, espera-se uma relação negativa com novas entradas. $\mathrm{Na}$ variável (ICtb2) intensidade de capital assume-se a possibilidade de uma relação não linear dado que resultados de estudos anteriores não são consensuais quanto à relação linear entre estas duas variáveis.

Tabela 3 - Variáveis de interação

\begin{tabular}{|c|c|c|c|c|}
\hline Variáveis de interação & Nome & Sinal & Definição & Fonte \\
\hline \multirow{2}{*}{$\begin{array}{l}\text { Produtividade do trabalho } \\
\text { vs Intensidade tecnológica }\end{array}$} & ITmPd & - & Intens_tec_med* logaritmo da produtividade & GPEARI/INE \\
\hline & ITbxPd & - & Intens_tec_bx* logaritmo produtividade & GPEARI/INE \\
\hline \multirow{2}{*}{$\begin{array}{l}\text { Intensidade capitalística vs } \\
\text { ciclo de vida da indústria }\end{array}$} & ICCi_ma & - & Imobilizado_corpóreo /trabalhador * Ciclo vida da indústria maduro & INE / QP \\
\hline & ICCi_cra) & $+/-$ & $\begin{array}{l}\text { Imobilizado corpóreo /trabalhador * Ciclo vida da indústria } \\
\text { crescimento }\end{array}$ & INE/QP \\
\hline Lucros vs IT_média & LitecM & + & $\begin{array}{l}\text { (Resultado liquido mais impostos/ /N_empresas)* Intensidade } \\
\text { tecnológica Média }\end{array}$ & INE/GPEARI \\
\hline Lucros vs IT_bx & LitecB & + & $\begin{array}{l}\text { (Resultado liquido mais impostos/ /N_empresas)* Intensidade } \\
\text { tecnológica_baixa }\end{array}$ & INE/GPEARI \\
\hline
\end{tabular}

Fonte: Autores. 
$\mathrm{Na}$ tabela 4 apresentam-se as variáveis cíclicas que controlam a sensibilidade das entradas relativamente às expectativas de benefícios da evolução cíclica da economia e da indústria.

Tabela 4 - Variáveis cíclicas

\begin{tabular}{|c|c|c|c|c|}
\hline \multicolumn{5}{|c|}{ Variáveis exógenas } \\
\hline Variáveis cíclicas & Nome & Sinal & Definição & Fonte \\
\hline \multirow{3}{*}{ Ciclo de vida da indústria } & Ciclo_Cresc (cicloC) & + & $\begin{array}{l}\text { Variável dicotómica (valor } 1 \text { quando o sector se encontra } \\
\text { na fase inicial e valor } 0 \text { para as outras fases) }\end{array}$ & GPEARI \\
\hline & $\begin{array}{l}\text { Ciclo_Inter } \\
\text { (cicloI) }\end{array}$ & $+/-$ & $\begin{array}{l}\text { Variável dicotómica (valor } 1 \text { quando o sector se encontra } \\
\text { na fase intermédia e valor } 0 \text { para as outras fases). }\end{array}$ & \multirow[t]{2}{*}{ GPEARI } \\
\hline & $\begin{array}{l}\text { Ciclo_mad. } \\
\text { (cicloM) }\end{array}$ & $+/-$ & $\begin{array}{l}\text { Variável dicotómica (valor } 1 \text { quando o sector se encontra } \\
\text { na fase intermédia e valor } 0 \text { para as outras fases) }\end{array}$ & \\
\hline $\begin{array}{l}\text { Crescimento do PIB da } \\
\text { economia }\end{array}$ & $\Delta \mathrm{PIB}(\mathrm{t}-1)$ & + & $\begin{array}{l}\text { Taxa interanual ex-ante do produto interno bruto (PIB), } \\
\text { calculado a preços constantes. }\end{array}$ & $\begin{array}{l}\text { Banco de } \\
\text { Portugal }\end{array}$ \\
\hline
\end{tabular}

\section{Resultados da estimação}

O resultado obtido para a estatística de Hausman permitiu, como já foi referido anteriormente, concluir que o modelo de efeitos fixos é o mais adequado para analisar os fatores explicativos da entrada de novas empresas. Assim, procedeu-se à estimação dos modelos utilizando a opção do software Stata vce (cluster CAE) cujo resultado se apresenta na tabela 5.

Tabela 5 - Resultados da estimação com dados em painel - modelo efeito fixos

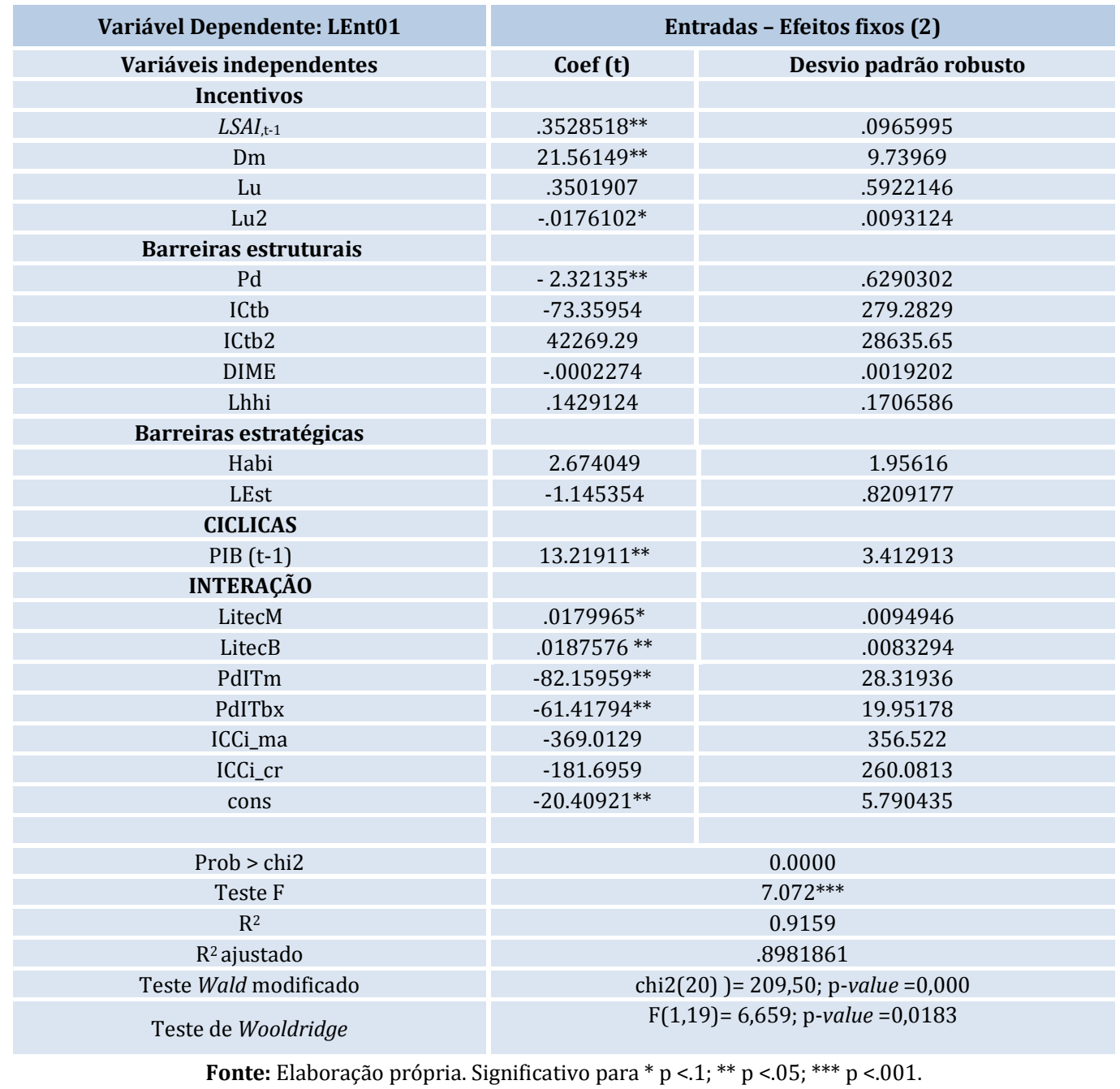

(a) A variáveis dummy ciclo-maduro (CicloM) e intensidade tecnológica baixa (ItecB) não entram na regressão para evitar uma colinearidade perfeita; (1) Efeitos Fixos com opção desvio padrão não ajustado - default; (2) Efeitos Fixos com opção desvio padrão ajustado (opção cluster robust). (b) Wooldridge (2002).

Os resultados obtidos permitem confirmar que globalmente todos os vetores, com exceção das barreiras estratégicas, contêm variáveis estatisticamente significativas na relação com a entrada de novas empresas.
No que respeita às variáveis que integram o vetor de incentivos três variáveis são importantes na determinação das entradas: saídas do período anterior $\left(\mathrm{LSAI}_{\mathrm{t}-1}\right)$ dimensão do mercado do período anterior (Dm) e lucro (Lu2). 


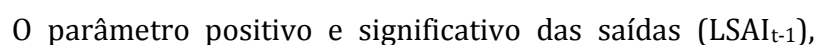
consistente com outros trabalhos empíricos, nomeadamente Andraz (2013) e Rosenbaum e Lamort (1992), mostra a grande significância da saída do período anterior na determinação da entrada. Esta relação confirma que o efeito de substituição é um fator determinante de novas entradas na indústria transformadora portuguesa. A correlação temporal de curto prazo entre a decisão de entrada e de saída também sugere que sectores com fluxos elevados de entrada, de uma forma geral, registam também elevados fluxos de saída. Note-se que, de acordo com os cálculos dos autores, a percentagem média de saída de empresas jovens (com menos de 3 anos de vida) da indústria no seu conjunto é de $20 \%$, revelando uma fraca capacidade de adaptação ao mercado das novas empresas e consequente elevada rotação empresarial.

Os resultados confirmam a hipótese formulada de que a dimensão do mercado do período anterior (Dm) é importante na decisão de entrada. Este resultado é consistente com a visão convencional de que os potenciais empreendedores ponderam a dimensão do mercado como um sinal de oportunidades de mercado, confirmado em diversos estudos (Mata 1993; Fotopoulos \& Spence, 1998; Barbosa, 2007).

As hipóteses formuladas para o efeito da variável lucro (lu) da indústria não parecem ser suportadas pelo resultado da estimação. Este resultado não é surpreendente, na medida em que os resultados empíricos sobre o efeito dos lucros sobre as entradas não são conclusivos. Porém, este estudo encontrou evidências de uma relação quadrática (na forma de "U" invertido) entre os lucros e a entrada de novas empresas. Este resultado sugere que o crescimento de entradas está associado a um maior nível de lucros nas fases iniciais, invertendo-se o processo a partir de um determinado ponto em que o aumento marginal das entradas decresce à medida que os lucros aumentam. Este resultado traduz a dinâmica da evolução dos mercados. Numa primeira fase os empreendedores potenciais são atraídos para o mercado em função das expectativas de lucros após a entrada. No entanto, à medida que o número de concorrentes aumenta, as empresas estabelecidas procuram cristalizar suas posições criando barreira. Por sua vez, as barreiras criadas dificultam ou limitam a entrada de novos concorrentes, pelo que há uma diminuição marginal de novas entradas e, simultaneamente, as margens de lucros por empresa aumentam.

Até à data, não se conhece na literatura empírica outro estudo que tenha testado a hipótese da não linearidade desta relação.

Relativamente às barreiras estruturais, os resultados mostram que um aumento de $1 \%$ na produtividade tem um impacto negativo sobre as entradas potenciais de $0.0232 \%$. O sinal negativo associado ao coeficiente desta variável, com um nível de significância de 5\%, corrobora as conclusões de outros estudos, designadamente Taymaz (1997), que sectores com níveis de produtividade elevados estão associados a fortes barreiras a novas entradas.
Quanto às variáveis cíclicas, os resultados sugerem que o fluxo de entradas de novas empresas é sensível à evolução ex- ante do ciclo económico. Este resultado confirma a tendência pró-cíclica de novas entradas, também observadas por outros autores, nomeadamente Mata (1996) e Geroski et al., (2007).

As variáveis de interação entre lucro (Lu) do sector e respetiva intensidade tecnológica LitecM e LitecB apresentam coeficientes positivos e estatisticamente significativos sugerindo que quando se considera o nível de intensidade tecnológica o lucro da indústria influência positivamente a entrada da indústria. Apesar da relação estatisticamente reduzida desta variável merece uma referência sobretudo pelo facto de não se encontrar na literatura empírica outro estudo onde esta relação tenha sido analisada.

Os coeficientes da estimação associados a estas variáveis de interação da produtividade do trabalho com a intensidade tecnológica média e baixa (PdITm e PdITbx) são, como se esperava, negativos e significativos, sugerindo que a produtividade, conforme resultado da estimação supraanalisada, também constitui uma barreira, tendo em conta a intensidade tecnológica do sector.

\section{Conclusão, considerações sobre políticas públicas e limitações do estudo}

Ao longo deste trabalho procurou-se analisar os fatores determinantes de entradas de empresas na indústria transformadora portuguesa, no contexto do processo de dinâmica empresarial.

Os resultados permitem identificar alguns fatores relevantes e sugerir algumas orientações sobre políticas públicas.

Verificou-se, tal como seria de esperar, que as entradas são sensíveis à dimensão de mercado. Os mercados de maior dimensão oferecem maiores possibilidades para que os potenciais produtores possam incorporar-se.

Este estudo encontrou evidências de uma relação quadrática (na forma de "U" invertido) entre os lucros e a entrada de novas empresas sugerindo que o incentivo lucro apenas é atrativo até determinado limiar. Tanto quanto tenhamos conhecimento não há na literatura empírica outro estudo que tenha apresentado este tipo de relação entre estas variáveis, pelo que consideramos ser um resultado pioneiro.

No que respeita às barreiras estruturais só a produtividade do trabalho parece condicionar novas entradas. Os custos irreversíveis, a concentração e economias de escala não são estatisticamente significativos no processo de entrada. Estes resultados parecem indicar que a entrada é bastante fácil e a saída também, por conseguinte as barreiras à entrada e saída são relativamente reduzidas. Nesta perspetiva, a reflexão de Geroski (1991) enquadra-se nesta análise e não podia ser mais apropriado para sintetizar uma das principais conclusões deste estudo: a entrada é fácil mas a penetração no mercado após a entrada, e até mesmo a sobrevivência, parece ser mais difícil. Todavia, o facto de as barreiras não serem significativas, não significa que não 
sejam importantes, no sentido de distribuir desigualmente o fluxo de entrada pelos vários sectores. Esta questão carece de um estudo mais aprofundado em trabalhos posteriores.

A forte correlação entre entradas e saídas, associada a barreiras à entrada pouco significativas na indústria transformadora portuguesa sugere uma elevada rotação das empresas, refletindo uma fraca capacidade das empresas de se adaptarem ao mercado, designadamente pela incapacidade de realizar os investimentos necessários em determinadas fases do seu crescimento.

Sendo a entrada um processo relativamente fácil, as orientações políticas deveriam ser direcionadas no sentido de estimular o crescimento sustentado das novas empresas. Desde logo, incentivando o vetor produtividade nas vertentes formação/qualificação dos recursos humanos, parcerias em I\&D e acesso a capital de risco.

A conjunção de políticas públicas de facilitação do acesso aos mercados de capitais, em condições de preço e quantidade adequados, com estratégias empresariais de investimento, orientadas para o crescimento, poderá contribuir para aumentar a dimensão média do tecido empresarial, criar economias de escala e estimular a inovação, contribuindo desta forma para melhorar os índices de competitividade da indústria.

Caberá às empresas ultrapassar as suas limitações de escala, criando parcerias estratégicas que permitam a dimensão necessária para aceder a mercados tão diversos como o de capitais, comerciais, tecnológicos, entre outros.

Este estudo tem algumas limitações designadamente a análise agregada a 2 dígitos (Divisão CAE) da indústria transformadora, que, de alguma forma, esconde a heterogeneidade dos sectores de atividade. 0 estudo de novas entradas e seus determinantes a um nível de maior desagregação da indústria fica para desenvolvimentos futuros na linha deste trabalho.

\section{Referências}

Acs, Z. J. \& Audretsch, D. B. (1989). Small-firm entry in U.S. manufacturing. Economica, 56 (222), 255-265.

Andraz, G. (2013). Determinantes da dinâmica empresarial. O caso da indústria transformadora Portuguesa. Tese de Doutoramento, Universidade de Évora, Évora.

Austin, J. S. \& Rosenbaum, D. I. (1990). The Determinants of entry and exit rates into U.S. manufacturing industries. Journal Review of Industrial Organization, 5(2), 211-223.

Bain, J. S. (1956). Barriers to new competition. Cambridge, MA: Harvard University Press.

Baldwin, J. (1995). The Dynamics of Industrial competition: A North American perspective. Cambridge: University Press.

Barbosa, N. (2007). An integrative model of firms' entry decisions. Applied Economics Quarterly, 53(1), 45-69.

Barney, J. (1991). Firm resources and sustained competitive advantage. Journal of Management Studies, 17(1), 99120.

Carreira, C. M. G. (2004). Dinâmica industrial e crescimento da produtividade: Uma análise macroeconómica do papel da aprendizagem e da reafectação de recursos no crescimento industrial. Tese de Doutoramento, Universidade de Coimbra, Coimbra.

Caves, R. E. \& Porter, M. E. (1976). Barriers to exit. In R. Masson \& e D. Qualls (eds.), Essays on Industrial organization in honor of Joe $S$. Bain (pp. 120-157). Cambridge: Ballinger Publishing Co.
Eurostat (2006). Online statistical databases of the European Commission's directorate general for statistics (EUROSTAT). Consultado em abil, 6, 2012, em http://ec.europa.eu/eurostat.

Fotopoulos, G. \& Spencer, N. (1998). Entry and exit from manufacturing industries: symmetry, turbulence and simultaneity Some empirical evidence from Greek manufacturing industries, 1982-1988. Applied Economics, 30 (2), 245-262.

Geroski, P. (1991). Market dynamics and entry. Oxford: Blackwell.

Geroski, P. (1991a). Reflections on the entry process. In P. Geroski \& J. Schwalbach (eds.), Entry and market contestability: an international comparison (pp. 282-296). Oxford: Basil Blackwell.

Geroski, P. (1995). What do we know about entry? International Journal of Industrial Organization, 13 (4), 421-440.

Geroski, P. A., Mata, J. \& Portugal, P. (2007). Founding conditions and the survival of new firms, DRUID Working Papers, Copenhagen Business School, Aalborg University.

Hsiao, C. (2003). Analysis of panel data. 2a. ed., New York: Cambridge University Press

Kessides, I. (1991). Entry and market contestability. The evidence from United States. In P. Geroski \& J. Schwalback (eds.), Entry and market constestability: an international comparison, 23-48. Oxford: Basil Blackwell.

Khemani, R. S. \& Shapiro D. M. (1987). The determinants of entry and exit reconsidered. International Journal of Industrial Organization, 5(1), 15-26.

Klepper, S. (1996). Entry, exit, growth, and innovation over the product life cycle. American Economic Review, 86(3), 562-583.

Lambson, V. E. (1991). Industry evolution with sunk costs and uncertain market conditions. International Journal of Industrial Organization, 9(2), 171.

Mata, J. (1991). Entrada e mobilidade. Um estudo da indústria transformadora portuguesa. Tese de Doutoramento, Universidade do Monho, Minho.

Mata, J. (1993). Firm entry and firm growth. Review of Industrial Organization, 8(5), 567-578.

Mata, J. (1996). Business conditions and business starts. International Journal of the Economics of Business, 3(3), 295-305.

Nelson, R. R. \&. Winter, S. G, (1982). An evolutionary theory of economic change. Cambridge, Mass: Harvard University Press.

Nystrom, K. (2007). Patterns and determinants of entry and exit in industrial sectors in Sweden. Journal International Entrepreneurship, 5 (3-4), 85-110.

Orr, D. (1974). The determinants of entry: a study of the Canadian manufacturing industries. Review of Economics and Statistics, 56(1), 58-66.

Rosenbaum, D. I. e F. Lamort (1992). Entry, barriers, exit, and sunk costs: an analysis. Applied Economics, 24(3), 297-304.

Schumpeter, J.A. (1935). The analysis of economic change, Review of Economic Statistics, 17 (4), 2-10.

Segarra, A. (2002). La entrada de empresas. In A. Segarra (ed.), La creación y la supervivencia de las empresas industriales (pp.55-72). Madrid: Civitas Ediciones.

Siegfried, J. J. \&. L. B. Evans (1994). Empirical studies of entry and exit: a survey of the evidence. Review of Industrial Organization, 9 (2), 121-155.

Taymaz, E. (1997). Small and medium-sized industry in Turkey. Technology Review, 80(7), 1-147.

Wagner, J. (1994). Small firm entry in manufacturing industries: Lower Saxony, 1979-1989. Small Business Economics, 6(3), 211-223.

Wooldridge, J. M. (2002). Econometric analysis of cross section and panel data. Cambridge: MIT Press.

Yip, G. S. (1982). Barriers to entry: A corporate perspective. Lexington, Lexicon Books.

Submetido: 14.05 .2014

Aceite: 28.11 .2014 